\section{Some Remarkable Properties of Diagonally Correlated MIMO Channels}

\author{
Hüseyin Özcelik and Claude Oestges
}

\begin{abstract}
This paper investigates so-called diagonally correlated multiple input-multiple output (MIMO) channels, which provide higher ergodic capacity than independent and identically distributed (i.i.d.) fading channels. The presented analysis details physical scenarios leading to such channels, some properties of the channel matrix, and an analytical expression for its ergodic capacity.
\end{abstract}

Index Terms-Capacity, correlation, Multiple-Input Multiple-Output (MIMO), polarization.

\section{INTRODUCTION}

It is well known that the ergodic multiple input-multiple output (MIMO) channel capacity grows asymptotically linearly with the number of antennas in a symmetric channel [1]. For Rayleigh-fading channels, it is usually believed that channel correlations always reduce capacity and that, therefore, independent identically distributed (i.i.d.) channels would yield maximum ergodic capacity. In the case of $n$ antennas at both link ends and disregarding bandwidth, the ergodic capacity of i.i.d. channels can be expressed as

$$
\bar{C}_{\text {iid }}=E\left\{\log _{2} \kappa\right\}=E\left\{\log _{2} \operatorname{det}\left(\mathbf{I}_{n}+\frac{\rho}{n} \mathbf{H} \mathbf{H}^{H}\right)\right\}
$$

where $E\{\cdot\}$ stands for the expectation operation, $\mathbf{I}_{n}$ denotes the $n \times n$ identity matrix, $\rho$ is the average receive SNR, $(\cdot)^{H}$ is Hermitian conjugation, and $\mathbf{H}$ is the normalized channel matrix, respectively.

It was recently shown in [2] and [3] that a simple mutual information metric equal to $E\{\kappa\}$ allows for analysis of ergodic MIMO capacity as a function of the correlations between different channel matrix elements. A most surprising result was that ergodic mutual information and capacity ${ }^{1}$ are not maximized by i.i.d. fading conditions but by channels with so-called high diagonal correlations. Are such channels realistic? Actually, there is so far no clear picture on what propagation scenarios that result in such channels look like. The characterization of such scenarios is the goal of this paper. We will also illustrate through analytical results that these diagonally correlated channels exhibit higher ergodic capacity ${ }^{2}$ than i.i.d. channels. Finally, we will introduce an approach on how to construct $n \times n$ diagonally correlated channels in general.

Manuscript received March 7, 2005; revised June 29, 2005. This work was supported by the European Network of Excellence NEWCOM (Network of Excellence in Wireless Communications). The work of C. Oestges was supported by Fonds National de la Recherche Scientifique (FNRS). The review of this paper was coordinated by Dr. K. Dandekar.

H. Özcelik is with the Institut für Nachrichtentechnik und Hochfrequenztechnik, Vienna, Austria (e-mail: hueseyin.oezcelik@tuwien.ac.at).

C. Oestges is with Fonds National de la Recherche Scientifique-FNRS, Microwave Laboratory, Université Catholique de Louvain, 1348 Louvain-laNeuve, Belgium (e-mail: oestges@emic.ucl.ac.be).

Digital Object Identifier 10.1109/TVT.2005.858193

${ }^{1}$ Equation (1) represents the capacity in the strict Shannon sense only for i.i.d. channels. For correlated channels, (1) is only the mutual information with equal power allocation, although it is often called capacity through a well-accepted abuse of language.

${ }^{2}$ This is true not only for the ergodic mutual information, but also for the ergodic capacity in the true Shannon sense (as the mutual information with equal power allocation is a capacity lower bound for non-i.i.d. channels).

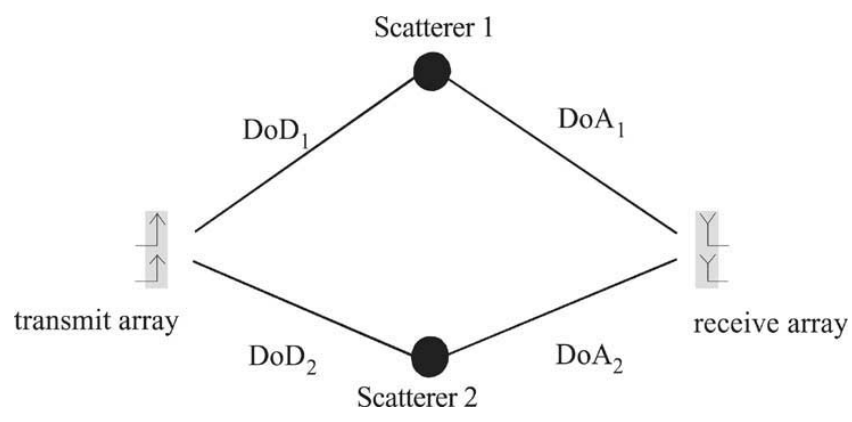

Fig. 1. Illustration of a $2 \times 2$ diagonally correlated channel.

\section{Diagonally CORRElated $2 \times 2$ MIMO ChanNElS}

\section{A. Channel Matrix and Covariance}

In the case of a $2 \times 2$ system, the channel matrix reads as

$$
\mathbf{H}=\left[\begin{array}{ll}
h_{11} & h_{12} \\
h_{21} & h_{22}
\end{array}\right] .
$$

From [3], it is clear that for $2 \times 2$ MIMO Rayleigh-fading channels, the capacity is maximized if the absolute value of the full MIMO correlation matrix $\mathbf{R}_{\mathbf{H}}=E\left\{\operatorname{vec}(\mathbf{H}) \operatorname{vec}(\mathbf{H})^{H}\right\}$, with vec $(\cdot)$ denoting the column-wise stacking of matrix elements into a column vector, fulfills

$$
\left|\mathbf{R}_{\mathbf{H}}\right|=\left[\begin{array}{llll}
1 & 0 & 0 & 1 \\
0 & 1 & 1 & 0 \\
0 & 1 & 1 & 0 \\
1 & 0 & 0 & 1
\end{array}\right]
$$

In (3), antenna amplitude correlations $E\left\{h_{i j} h_{l j}^{*}\right\}$ (receive correlation when transmitting from antenna $j$ ) and $E\left\{h_{i j} h_{i l}^{*}\right\}$ (transmit correlation when receiving on antenna $i)$ are equal to $0\left(i \neq j \neq l=1,2 ;(\cdot)^{*}\right.$ denotes complex conjugate). Simultaneously, the remaining entries, which we define as diagonal correlations, are equal to unity. Such channels are defined as $2 \times 2$ diagonally correlated. Henceforth, the amplitudes of the matrix elements of a $2 \times 2$ diagonally correlated channel read as

$$
\left|\mathbf{H}_{\text {diag }}\right|=\left[\begin{array}{ll}
\left|h_{11}\right| & \left|h_{12}\right| \\
\left|h_{12}\right| & \left|h_{11}\right|
\end{array}\right]
$$

with $E\left\{h_{11} h_{12}^{*}\right\}=0$.

\section{B. Physical Scenarios}

A first illustration of a diagonally correlated $2 \times 2$ MIMO channel is shown in Fig. 1. Such a channel occurs in scenarios in which only two, nearly equally powered, independent Rayleigh-fading multipath components with orthogonal directions-of-arrival (DoAs) and directions-of-departure (DoDs) exist. It is easy to observe that if a single DoD couples into a single DoA and vice versa, then the diagonally correlated channel results [4]. Note that the orthogonality of directions is a sufficient condition but is not necessary. It is required that the corresponding steering/response vectors are orthogonal. Clearly, a fixed wireless access channel might occasionally be diagonally correlated (i.e., for a given base station, at any subscriber's location where there are no more than two major scatterers, with orthogonal DoDs and DoAs at both link ends). Yet, it must be acknowledged that this situation is by no means a very common situation. However, it can be shown that $2 \times 2$ dual-polarized links constitute a highly practical scenario offering the diagonal correlation structure of (3). Indeed, it has been illustrated 
in [5] that a dual-polarized channel (assuming collocated antennas at both link ends) can be represented by the following matrix:

$$
\mathbf{H} \approx \alpha\left[\begin{array}{cc}
1 & \mu \chi \mathrm{e}^{j \phi} \\
\chi \mathrm{e}^{j \phi} & \mu
\end{array}\right]
$$

where $\alpha$ is a random time-varying fading variable, $\phi$ is a random time-varying angle uniformly distributed over $[0,2 \pi]$, and $\mu$ and $\chi \in$ $[0,1]$ are random time-varying variables that represent respectively the ratio of the propagation loss in orthogonal components and the crosspolar discrimination (XPD). In slanted schemes (antennas oriented along $\pm 45^{\circ}$ ), it is evident that $\mu=1$ due to the array symmetry. For horizontal/vertical (HV) schemes, the average value of $\mu$ depends on the polarization selectivity of the environment. However, it is generally assumed that $\mu \approx 1$ in most HV scenarios. Furthermore, experimental results [6] suggest that, for large receive-to-transmit distances or in large excess path-loss areas, $\alpha$ is Rayleigh distributed (the Ricean $\mathrm{K}$ factor is very low) and that $\chi \approx 1$ (the XPD is about $0 \mathrm{~dB}$ ). Note that since the K-factor and the XPD are strongly correlated [6], the Rayleigh behavior might be a sufficient condition to obtain both a low K-factor and $\chi \approx 1$. So, in these cases, the dual-polarized channel matrix reduces to

$$
\mathbf{H} \approx \alpha\left[\begin{array}{cc}
1 & \mathrm{e}^{j \phi} \\
\mathrm{e}^{j \phi} & 1
\end{array}\right] .
$$

In (6), the matrix elements are Rayleigh fading. The receive and transmit antenna correlations are also equal to 0 , owing to the random phase shift between co- and cross-polar components $\left(E\left\{e^{j \phi}\right\}=0\right)$. By contrast, the diagonal correlations are both equal to 1 . This exactly fulfills the requirement of (3).

Finally, it is worth noting that the formalism of the finite scatterer model [7] or the approach presented by [8] allows the diagonally correlated channel $\mathbf{H}_{\text {diag }}$ to be expressed as

$$
\mathbf{H}_{\mathrm{diag}}=\mathbf{A}_{\mathrm{Rx}}(\mathbf{D} \circ \mathbf{G}) \mathbf{A}_{\mathrm{Tx}}^{T} .
$$

Here, $(\cdot)^{T}$ denotes transposition, is the element-wise Schur-Hadamard multiplication, while $\mathbf{G}$ is a $2 \times 2$ i.i.d. complex Gaussian randomfading matrix. The two columns of the unitary matrix $\mathbf{A}_{\mathrm{Tx}}\left(\mathbf{A}_{\mathrm{Rx}}\right)$ constitute orthogonal steering (response) vectors of DoDs (DoAs). The $2 \times 2$ coupling matrix $\mathbf{D}$ is either a diagonal or antidiagonal matrix with identical entries, producing equally powered, one-to-one coupling between DoDs and DoAs. By contrast, the popular Kronecker model is not able to represent diagonally correlated channels.

\section{Ergodic and Outage Capacity}

A closer look at (7) allows the distribution of the singular values of $\mathbf{H}_{\text {diag }}$ to be derived. The orthogonal steering vectors of the unitary receive (transmit) matrix $\mathbf{A}_{\mathrm{Rx}}\left(\mathbf{A}_{\mathrm{Tx}}\right)$ constitute the left (right) singular vectors, except for a constant phase term. The magnitudes of the singular values of $\mathbf{H}_{\mathrm{diag}},\left|s_{i}\right|$, are given by the magnitudes of the complex-Gaussian-distributed, nonzero elements of $(\mathbf{D} \circ \mathbf{G})$. Hence, $\left|s_{i}\right|$ follows a Rayleigh distribution. As a consequence, the eigenvalues $\lambda_{i}=\left|s_{i}\right|^{2}$ of the Gramian $\mathbf{H}_{\mathrm{diag}} \mathbf{H}_{\mathrm{diag}}^{H}$ are chi-squared distributed with two degrees of freedom. Since we normalize to unity the average power of each channel matrix coefficient, the Rayleigh parameter of the distribution of $\left|s_{i}\right|$ becomes unity, too. The ergodic capacity in a $2 \times 2$ diagonal channel is then exactly given by

$$
\begin{aligned}
\bar{C} & =E\left\{\sum_{k=1}^{2} \log _{2}\left(1+\frac{\rho}{2} x^{2}\right)\right\} \\
& =2 \cdot E\left\{\log _{2}\left(1+\frac{\rho}{2} x^{2}\right)\right\}
\end{aligned}
$$

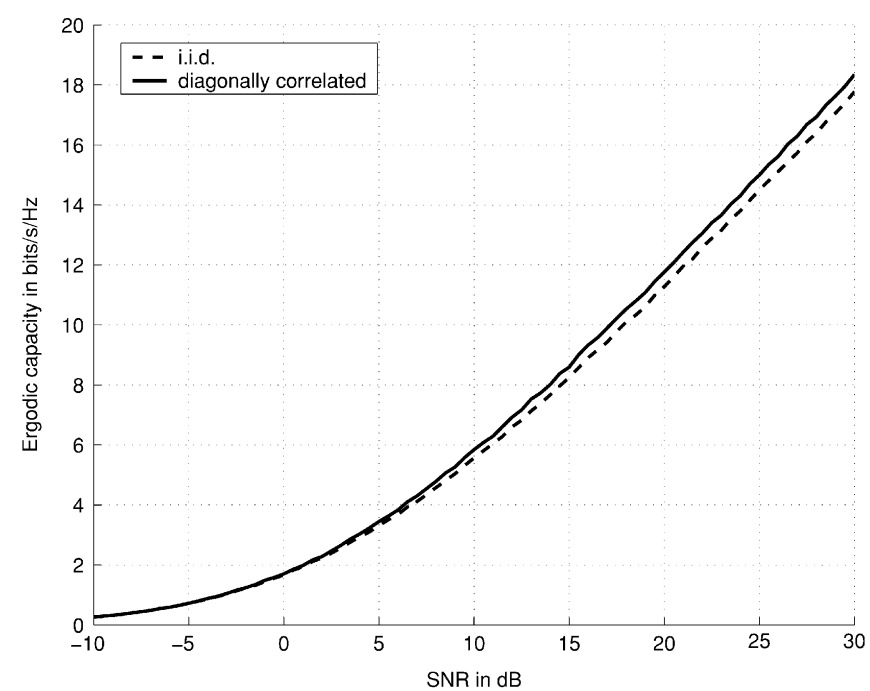

Fig. 2. Ergodic capacity of diagonally correlated and i.i.d. channels as a function of SNR.

$$
\begin{aligned}
& =2 \int_{0}^{\infty} \log _{2}\left(1+\frac{\rho}{2} x^{2}\right) x e^{-\frac{x^{2}}{2}} d x \\
& =2 \log _{2}(e) e^{\frac{1}{\rho}} \mathrm{E}_{1}\left(\frac{1}{\rho}\right)
\end{aligned}
$$

where $\mathrm{E}_{1}(z)$ is the En-function [9] for $n=1$ that satisfies $\mathrm{E}_{1}(z)=\int_{1}^{\infty}\left(e^{-t z} / t\right) d z$.

Close scrutiny of (8) reveals that the diagonally correlated channel exhibits a higher ergodic capacity than the corresponding i.i.d. channel, irrespective of the SNR $\rho$. Note also that the low-SNR behavior is similar to the high-SNR behavior. It should be remembered that correlations are already specified via (3), so that (8) does not exhibit any dependence toward correlation coefficients.

In Fig. 2, the ergodic capacity is plotted as a function of SNR. The capacity advantage of diagonally correlated channels is also confirmed by dual-polarized experimental results [6] in large excess path-loss conditions (note that the capacity results in [6] are normalized to remove any path-loss effect on the SNR, so that the comparison with i.i.d. channels using such normalization is fair and similar to the normalization used to derive the analytical result). Finally, we performed Monte Carlo simulations with $5 \cdot 10^{5}$ realizations each to show the capacity cumulative distribution functions (CDFs) of both channels at a given SNR of $20 \mathrm{~dB}$ (Fig. 3). Interestingly, while the ergodic capacity of the diagonally correlated channel is larger than the i.i.d. counterpart, the CDF presents a flatter slope than that of the i.i.d. channel; i.e., its outage capacity is lower for outage levels less than approximately $15 \%$.

An intuitively appealing reason for this capacity behavior is as follows. The channel amplitudes $\left|h_{11}\right|$ and $\left|h_{22}\right|=\left|h_{11}\right|$ in (4) fade strictly in tandem, and so do the off-diagonal elements, but these pairs fade independent of each other, providing two perfectly separated virtual subchannels on their own. This improved separation of thesubchannels results in a higher ergodic capacity. Since the degrees of freedom reduce from four to two, diversity in diagonally correlated channels is lower than for i.i.d., leading to a flatter slope of the capacity CDF.

\section{Diagonally CORRELATED $n \times n$ MIMO CHANNELS}

The concept of the $2 \times 2$ diagonally correlated channel can be generalized as follows. Consider a symmetric $n \times n$ MIMO channel with $n$ orthogonal DoDs and $n$ orthogonal DoAs. We call such a channel 


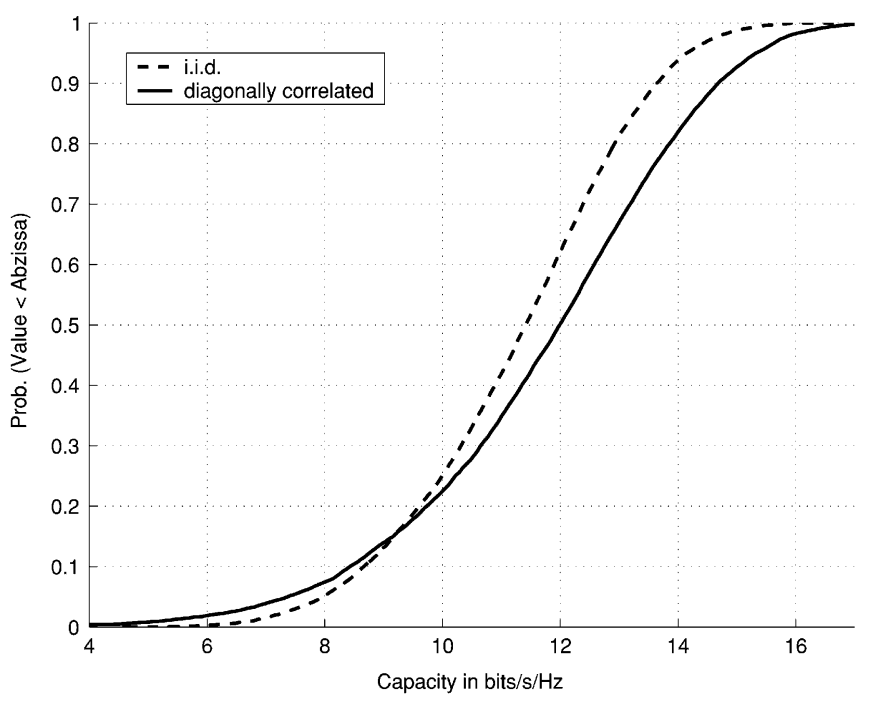

Fig. 3. Capacity CDF of a $2 \times 2$ i.i.d. and diagonally correlated channel at 20 $\mathrm{dB}$ receive $\mathrm{SNR}$.

diagonally correlated if each single DoD couples into a single DoA and vice versa. The analytical modeling stays the same and follows (5). Of course, the dimensions of the unitary steering and response matrices $\mathbf{A}_{\mathrm{Tx}}$ and $\mathbf{A}_{\mathrm{Rx}}$, the coupling matrix $\mathbf{D}$, and the random fading matrix $\mathbf{G}$ must change to $n \times n$. Further, $\mathbf{D}$ is a sparse matrix with only one entry in each row and column, producing the needed one-to-one coupling. The entries of $\mathbf{D}$ have to be identical. The actual shape of $\mathbf{D}$ determines the appearance of the channel matrix and therefore the full correlation matrix. Interestingly enough, the amplitudes of the corresponding channel matrix entries exhibit a specific symmetry. It can be shown that the rows have to be cyclically shifted, either left [as in (7)] or right. As an example for $3 \times 3$ MIMO, the amplitudes of the channel matrix may result, for a given choice of $\mathbf{D}$, in

$$
\left|\mathbf{H}_{\text {diag }}\right|=\left[\begin{array}{lll}
\left|h_{11}\right| & \left|h_{12}\right| & \left|h_{13}\right| \\
\left|h_{12}\right| & \left|h_{13}\right| & \left|h_{11}\right| \\
\left|h_{13}\right| & \left|h_{11}\right| & \left|h_{12}\right|
\end{array}\right] .
$$

This would also correspond to, e.g., tri-polarized schemes (using three orthogonal polarizations at both the transmit and the receive sides).

Similarly to the $2 \times 2$ case, the eigenvalues $\lambda_{i}=\left|s_{i}\right|^{2}$ of the Gramian $\mathbf{H}_{\text {diag }} \mathbf{H}_{\text {diag }}^{H}$ are still independently identical chi-squared distributed with two degrees of freedom, independent of the actual shape of $\mathbf{D}$. If the channel is normalized to unity average power of each channel matrix coefficient, the variance of the Rayleigh-distributed $\left|s_{i}\right|$ increases linearly with the number of antennas. For $n$ antennas at both link ends, it equals $(n / 2)$. Hence, the ergodic capacity of an $n \times n$ diagonal channel is exactly given by

$$
\bar{C}=n \log _{2}(e) e^{\frac{1}{\rho}} \mathrm{E}_{1}\left(\frac{1}{\rho}\right) .
$$

In the above expression, the capacity only depends on the SNR and on the number of antennas (as the covariance matrix of the channel is fully determined through the diagonal-correlation assumption). Interestingly, the capacity grows exactly linearly with the number of antennas whereas the capacity of i.i.d. channels grows in $n$ only asymptotically.

\section{CONCLUSION}

This letter has proved that diagonally correlated channels have the remarkable property of providing higher ergodic capacity than i.i.d. channels but lower outage capacity at low outage levels. The study also answers which propagation scenarios would allow for such behavior to take place. An important class of diagonally correlated channels is constituted by dual-polarized Rayleigh-fading channels. We also show that, mathematically, the rows of the amplitudes of the channel coefficients have to be cyclically shifted versions of each other. Analytical formulas of ergodic capacity of symmetric $n \times n$ MIMO systems, $n$ arbitrary, have finally been detailed.

\section{ACKNOWLEDGMENT}

The authors heartily thank Prof. E. Bonek for his critical reading and helpful comments.

\section{REFERENCES}

[1] I. E. Telatar, "Capacity of multi-antenna Gaussian channels," Eur. Trans. Telecommun., vol. 10, no. 6, pp. 585-595, 1999.

[2] C. Oestges, B. Clerckx, D. Vanhoenacker-Janvier, and A. J. Paulraj, "Impact of fading correlations on MIMO communication systems in geometry-based statistical channel models," IEEE Trans. Wireless Commun., vol. 4, no. 3, pp. 1112-1120, May 2005.

[3] C. Oestges and A. J. Paulraj, "Beneficial impact of channel correlations on MIMO capacity," Electron. Lett., vol. 40, no. 10, pp. 606-607, 2004

[4] H. Özcelik, "Indoor MIMO channel models," Ph.D. dissertation, Techn. Univ. Wien, Vienna, Austria, 2005.

[5] C. Oestges, V. Erceg, and A. J. Paulraj, "Propagation modeling of multipolarized MIMO fixed wireless channels," IEEE Trans. Veh. Technol., vol. 53, no. 3, pp. 644-654, May 2004.

[6] V. Erceg, P. Soma, D. S. Baum, and S. Catreux, "Multiple-input multipleoutput fixed wireless radio channel measurements and modeling using dual-polarized antennas at $2.5 \mathrm{GHz}$," IEEE Trans. Wireless Commun., vol. 3, no. 6, pp. 2288-2298, Nov. 2004.

[7] A. G. Burr, "Capacity bounds and estimates for the finite scatterers MIMO wireless channel," IEEE J. Select. Areas Commun., vol. 21, no. 5, pp. 812 818, Jun. 2003

[8] A. M. Sayeed, "Deconstructing multi-antenna fading channels," IEEE Trans. Signal Process., vol. 50, no. 10, pp. 2563-2579, Oct. 2002.

[9] M. Abramowitz A. Stegun "Exponential integral and related functions," in Handbook of Mathematical Functions with Formulas, Graphs, and Mathematical Tables, New York: Dover, 1972 ch. 5. 